\title{
Dose Distribution in Semi-Field Radiotherapy Options for Upper and Middle Esophageal Cancer Radiotherapy
}

\author{
Biao Zeng ${ }^{1,2}$, Jiutang Zhang ${ }^{1,2}$, Jianhui Liu ${ }^{3}$, Hui Wang ${ }^{1,2^{*}}$, Qianxi Ni ${ }^{1,2}$ \\ ${ }^{1}$ Department of Radiation Oncology, Hunan Cancer Hospital \& the Affiliated \\ Cancer Hospital of Xiangya Medicine, Central South University, Changsha, \\ Hunan 410013, P.R. China; \\ ${ }^{2}$ Key Laboratory of Translational Radiation Oncology, Changsha, Hunan \\ 410013, P.R. China; \\ ${ }^{3}$ Department of Information, Hunan Hospital of Armed Police Forces, \\ Changsha, Hunan 410006, P.R. China.
}

First Author: Biao Zeng, Hunan Cancer Hospital radiotherapy physicist. Email: zengbiao008@163.com.

${ }^{*}$ Corresponding Author: Hui Wang, Doctoral Supervisor in Thoracic Radiotherapy, Department of Radiation Oncology, Hunan Cancer Hospital. Email: wanghui710327@163.com.

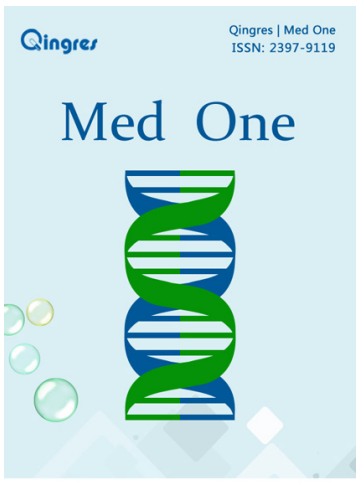

http://mo.qingres.com

\section{GOPEN ACCESS}

DOI: 10.20900/mo.201600014

Received: May 8, 2016

Accepted: July 3, 2016

Published: August 25, 2016

Copyright: ๑2016 Cain et al. This is an open access article distributed under the terms of the Creative Commons Attribution License, which permits unrestricted use, distribution, and reproduction in any medium, provided the original author and source are credited.

\begin{abstract}
Objective: To explore an adequate treatment method and provide a better irradiation mode for upper, and mid, esophageal carcinoma radiotherapy by comparing the dose distributions of six different semifield radiotherapy options.

Methods: Twenty patients with upper, and mid, esophageal carcinoma were selected for six treatment options, three [Options D-F] were semi-field irradiation: Option A-conventional 5 -field three-dimensional conformal radiation therapy (3D-CRT); option B-conventional 5-field intensity modulated radiation therapy (IMRT); option C-conventional 3-field IMRT; option D-5-field IMRT (upper target) and 3-field conformal radiation therapy (lower target); option E-5-field IMRT (upper target) and 3- field IMRT (lower target); and, option F-5-field IMRT (upper target) and 5-field IMRT (lower target). Dose distribution in the target areas and dose distribution in normal tissue were compared for the six options.
\end{abstract}

Results: Target coverage by the semi-field techniques was effective in a comparison of the six options. Lung low-dose area and mean lung dose (MLD) were lower in Options D and E. Option E conformal index 
in was higher than Option D.

Conclusion: Options $D$ and $E$ appear to be two relatively advantageous options as they allow effective delivery of the prescribed dose to the target volumes while minimizing the lung tissue exposure to low doses.

Key Words: Dose; IMRT; Semi-field; Esophageal cancer

\section{INTRODUCTION}

The radiotherapy target for upper, and middle, esophageal carcinoma spans the thoracic inlet. Anatomical characteristics cause target depths to vary significantly, resulting in uneven target dose distributions in radiotherapy and excessive exposure dose in normal tissues, especially the lungs. It is difficult for three-dimension-conformal radiotherapy (3D-CRT) to avoid vital organs, such as the spinal cord and lungs, while at the same time improving the target dose. Highly conformal radiotherapy techniques may improve target dose, but may cause excessive pulmonary tissue irradiations in low-dose patients. Therefore, to decrease the area of lungs exposed to low dosages is a physicist's primary consideration in developing radiotherapy plans. This study attempted to decrease lung tissue low-dose exposure areas and reduce the incidence of radiation pneumonitis by using a semi-field IMRT technique. It is a new technique for esophageal cancer treatment that has not yet, to our knowledge, been described in the literature. It is a semi-field, intensity-modulated mode for the upper half segment, and a 3-field 3D-CRT, or semi-field intensity-modulated mode for lower half segment. The aim is to provide a theoretical foundation for applying mono-isocenter upper and lower semi-field techniques via a dose comparison of different radiotherapy planning techniques.

\section{MATERIALS AND METHODS}

\subsection{Clinical Data}

Twenty patients with upper, and mid, esophageal cancer were selected, including 15 male and 5 female patients, 38-72 years old, with a mean age of 45 . Selection criteria: target ranged in esophagus tumor and lymphatic drainage area of thoracic upper and middle segments ${ }^{[1]}$.

\subsection{Methods}

\subsubsection{Delineation of Targets}

Gross tumor volume (GTV) on computed tomography (CT) planning is defined as the tumor extension (GTVt) and lymphadenopathies (GTVnd) that can be seen via diagnosis, staging imagology, and endoscopy. Clinical target volume (CTV) includes GTVt and GTVnd plus the lymphatic drainage area of subclinical risk, a margin of $1 \mathrm{~cm}$ around the GTVt and GTVnd, and $4 \mathrm{~cm}$ vertical of the GTVt or $2 \mathrm{~cm}$ at CT level with a lymphatic metastasis (GTVnd). It also includes a lymphatic drainage area with a higher lymphatic metastasis rate. CTV was edited to exclude structures such as vertebrae, without subclinical risk. Posteriorly, it defined the planning target volume (PTV) by adding a margin of $0.5 \mathrm{~cm}$ to the CTV.

\subsubsection{Treatment Planning}

CT scans was performed, during immobilization, of both chest and neck in a CT simulator. Physicians viewed the scanned images in order to identify tumor target areas.

A XiO (Elekta Ltd.) treatment planning system, Varian 600CD linear accelerator (Varian Medical Systems, Inc.) and 6 MV X rays were used. Six treatment options were developed for each patient, three conventional total-field and three semi-field plans. The conventional total-field options were: Option A (conventional 5-field 3D-CRT); Option B (conventional 5-field intensity modulated radiation therapy (IMRT)); and Option C (conventional 3-field IMRT). The mono-isocenter semi-field plan options were: Option D (5 semi-field IMRT in the upper target and 3 semi-field 3D-CRT in the lower target area); Option E (5 semi-field IMRT in the upper target and 3 semi-field IMRT in the lower target area); and, Option F (5 semi-field IMRT in both the upper and lower target areas).

Conformal plans used a coplanar design. IMRT plans used step-and-shoot delivery. All 5-and 3-field IMRT plans employed an equivalent split-field technique. Prescription dose to PTV was $60 \mathrm{~Gy}$ in 30 fractions. PTV was required to reach $100 \%$ of 60 Gy. A prescription dose should cover $95 \%$ of the 
PTV, with a maximum dose of $110 \%$. A prescription dose greater than $110 \%$ should not exist outside the target area.

No PTV cold spots and no hot esophagus wall hot spots were included in the PTV. The maximum dose to spinal cord was less than 42 Gy, lung V20 (lung volume receiving $\geq 20 \mathrm{~Gy}$ ) was less than 28 $\%$. Doses were all calculated using a superposition/ convolution algorithm.

\subsection{Program Evaluation}

Dose volume histograms were used to count: 1) dose to organs at risk; 2) dose indicators of PTV; 3) D90 (dose delivered to $90 \%$ of the PTV); 4) D95; 5) D100, maximum dose; 6) V100 (percent of the PTV receiving $100 \%$ of the prescribed dose); 7) V110, homogeneity index (HI); and, 8) conformity index $(\mathrm{Cl}) . \mathrm{Cl}^{[2]}=\frac{\mathrm{V} 100(\text { Target })}{\mathrm{V} 100 \text { (Body) }} \times \frac{\mathrm{V} 100(\text { Target })}{\mathrm{V} \text { (Target) }}$, where V100(Target) is the PTV covered by an isodose of $100 \%$, the $\mathrm{V} 100$ (Body) is the body volume covered by an isodose of $100 \%$, and V(Target) is the PTV.

$\mathrm{HI}$ was defined by D2/D98. A greater HI value indicates poorer homogeneity. As the $\mathrm{C} 1$ approaches 1 , conformation of the plan improves. $\mathrm{A} \mathrm{Cl}$ greater than 1 indicates that the irradiated volume (IV) is greater than the target volume (PTV) and healthy tissues are included. If $\mathrm{Cl}$ is less than 1, the target volume is only partially irradiated. After attempting to meet dose constraints to the organs at risk, lung volumes V5, V10, V20, and V30, and the mean lung dose (MLD) were compared for the six treatment plans.

\subsection{Data Analysis}

A one-way variance analysis was performed to compare the mean values of the six options using International Business Machines Corporation software SPSS (Statistical Package for the Social Sciences) 13.0. Statistical significance is defined as $p<0.05$.

\section{RESULTS}

\subsection{Various PTV Parameters Compared}

A data analysis shows that D100, D95, and D90 of conventional conformal Option A were all lower than the other five options. Target area coverage was poorer. There were no differences among the other five options. HI studies showed that conventional 5-(Option B) and 3-field IMRT (Option C) homogeneities were superior to conventional 3D-CRT option (Option A) or the three singleisocenter upper/mid semi-field radiotherapy options (Options D, E, and F). Cl studies showed that conventional 5-field IMRT (Option B), monoisocenter upper 5 semi-field and lower 3 semi-field IMRT (Option E), and, 5 semi-field IMRT in both the upper and lower target area (Option F), were more conformal than the other three options. The IV studies of the six options showed that the IV of patients with more than $3000 \mathrm{~cm} 3$ in Option C conventional 3-field IMRT option was the highest. The detailed data are in Table 1.

Table 1. PTV Parameters Compared

\begin{tabular}{lcccccc}
\hline $\begin{array}{l}\text { Treatment } \\
\text { Option }\end{array}$ & D100 (cGy) & D95 (cGy) & D90 (cGy) & Cl & HI & IV (cm3) \\
\hline Option A & $5440 \pm 212$ & $5714 \pm 132$ & $5809 \pm 118$ & $0.54 \pm 0.1$ & $1.10 \pm 0.02$ & $2280 \pm 290$ \\
Option B & $5590 \pm 275$ & $5880 \pm 125$ & $5960 \pm 135$ & $0.65 \pm 0.1$ & $1.09 \pm 0.02$ & $2270 \pm 280$ \\
Option C & $5562 \pm 256$ & $5893 \pm 143$ & $5968 \pm 142$ & $0.64 \pm 0.1$ & $1.09 \pm 0.02$ & $3210 \pm 260$ \\
Option D & $5566 \pm 231$ & $5843 \pm 127$ & $5991 \pm 112$ & $0.54 \pm 0.1$ & $1.15 \pm 0.02$ & $2305 \pm 292$ \\
Option E & $5514 \pm 221$ & $5792 \pm 104$ & $5929 \pm 142$ & $0.64 \pm 0.1$ & $1.16 \pm 0.02$ & $2314 \pm 260$ \\
Option F & $5522 \pm 198$ & $5823 \pm 124$ & $5956 \pm 134$ & $0.65 \pm 0.1$ & $1.15 \pm 0.02$ & $2290 \pm 289$ \\
F Value & 1.048 & 5.356 & 4.941 & 5.920 & 55.333 & 36.213 \\
$p$ Value & 0.393 & 0.000 & 0.000 & 0.000 & 0.000 & 0.000 \\
\hline
\end{tabular}

Terms: Cl: conformity index; Dx: dose delivered to $\mathrm{x} \%$ of the PTV; $\mathrm{F}$ Value: variance of the group of means; $\mathrm{HI}$ : homogeneity index; IV: irradiated volume; PTV: planning target volume; $p$ Value: calculated probability to determine the statistical significance of the hypothesis test. 


\subsection{Comparison of Radiation Dose of Lung Tissues}

In Option A (5-field 3D-CRT), Option B (5-field IMRT), and Option F (upper/lower mono-isocenter 5 semi-field IMRT), the low-dose area volume received by lung tissues was large. V20 and V30 did not increase as the number of fields increased. There was no statistical difference between the options. (Table 2). Coronal dose distributions of Options D, F, and $\mathrm{C}$ receiving 500 cGy appear in Fig. 1.

Table 2. Lung Tissue Radiation Dosages Compared

\begin{tabular}{lccccc}
\hline $\begin{array}{l}\text { Treatment } \\
\text { Option }\end{array}$ & V30 (\%) & V20 (\%) & V10 (\%) & V5 (\%) & MLD(Gy) \\
\hline Option A & $11.2 \pm 4.1$ & $30.8 \pm 1.5$ & $48.3 \pm 3.8$ & $60.5 \pm 6.2$ & $14.0 \pm 2.1$ \\
Option B & $12.8 \pm 3.8$ & $28.2 \pm 1.6$ & $48.7 \pm 3.6$ & $61.5 \pm 6.3$ & $13.9 \pm 2.1$ \\
Option C & $18.1 \pm 3.5$ & $28.5 \pm 1.9$ & $45.2 \pm 3.1$ & $56.5 \pm 6.1$ & $14.2 \pm 2.1$ \\
Option D & $13.3 \pm 4.2$ & $27.7 \pm 2.2$ & $44.9 \pm 3.3$ & $57.2 \pm 6.0$ & $12.7 \pm 2.1$ \\
Option E & $13.8 \pm 4.3$ & $28.3 \pm 2.2$ & $45.3 \pm 3.4$ & $57.5 \pm 6.4$ & $12.6 \pm 2.0$ \\
Option F & $13.2 \pm 3.9$ & $28.9 \pm 2.3$ & $48.1 \pm 3.1$ & $61.2 \pm 6.2$ & $14.2 \pm 2.1$ \\
F Value & 6.790 & 4.711 & 5.544 & 2.606 & 2.561 \\
$p$ Value & 0.000 & 0.001 & 0.000 & 0.029 & 0.031 \\
\hline
\end{tabular}

Terms: F Value: variance of the group of means; MLD: mean lung dose; PTV: planning target volume; $p$ Value: calculated probability to determine the statistical significance of the hypothesis test; Vx: lung volume receiving $\geq x \mathrm{~Gy}$.

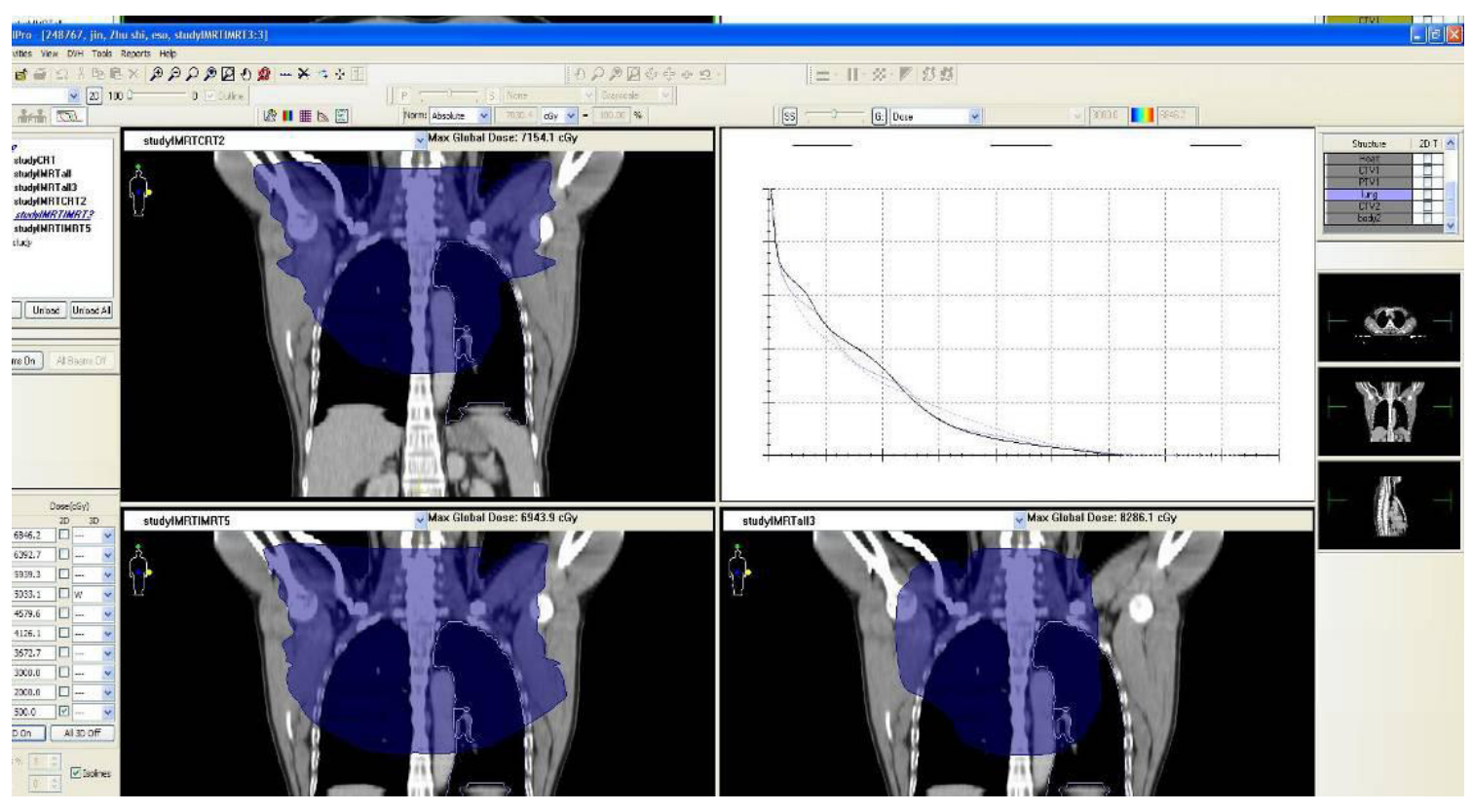

Fig. 1 Coronal dose distribution of Options D, F, and C in lung tissue receiving 500cGy 


\subsection{Segment Number and Treatment Monitor Unit (Mu) Comparison}

The number of segments and the treatment MU of Options $\mathrm{E}$ and $\mathrm{F}$ were significantly greater than the other options. It had fewer segments and its MU was the shortest. There were fewer Option $C$ segments than Options B, D, E, or F. There were no statistical differences between Option C, B, or D MU.

\section{DISCUSSION}

Upper-and-middle thoracic esophageal carcinoma radiotherapy aims to maximally increase doses to the target area to kill the tumor cells while lessening, or avoiding, radiating surrounding normal tissues and organs ${ }^{[3]}$. Studies have shown that, compared to conventional radiotherapy, 3D-CRT has the advantages of increasing target areas doses while protecting spinal cord and lung tissues. IMRT can provide more conformal, and even, dose distribution to tumor target areas and the V20 of the lung was also significantly lower than with 3D-CRT. Lung V5 (a low-dose area) was higher than with 3D -CRT ${ }^{[4]}$.

Chen et al. ${ }^{[5]}$ compared intensity modulated arc therapy (IMAT) and IMRT for esophageal carcinoma and concluded that IMAT could reach dosimetry requirements similar to IMRT, but had no advantage in protecting lung tissue. For this reason, using mono-isocenter upper, and lower semi-field, radiotherapy on upper, and middle, esophageal carcinoma was studied concerning aspects of the target area coverage, conformation, homogeneity, treatment time, and normal tissues protection, especially lung tissues, by using a mono-isocenter 5 semi-field IMRT technique for the upper segment and a 3 semi-field 3D-CRT, or a 3 or 5 semi-field IMRT technique, for the lower segment of the thoracic esophagus.

Research shows that the three treatment options using mono-isocenter upper and lower semifield radiotherapy obtained good results in target area coverage and could meet clinical requirements. Conformation was also but homogeneity was slightly poorer and it could protect normal tissue, especially lung tissue.

Lungs are relatively sensitive to radiation. Acute radiation pneumonitis and pulmonary fibrosis are the most common lung complications from radiotherapy of upper, and middle, thoracic esophageal carcinoma and are important factors affecting patient quality of life. Lung tissue dosages are also a major factor constraining the delivery of high doses to a target area in radiotherapy. With the growth of the incidence of esophageal carcinoma and the extensive development of radiotherapy, radio-pulmonary lesion treatment is increasingly important. Graham et al. ${ }^{[6]}$ argued that the incidence, and severity, of radiation pneumonitis is closely associated with radiation volume and lung tissue dosages. In a multivariate analysis, MLD, V20, and V30 were the proven to be the parameters most closely related to radiationinduced lung injury ${ }^{[6]}$. Wang et al. ${ }^{[7]}$ noted that V5 might be the most valuable predictive indicator for radiation-induced lung injury. As V $5>55 \%$, the incidence of grade 2, or greater, radiation-induced lung injuries might significantly increase ${ }^{[7]}$. Recent studies show that radiation pneumonitis incidence increases as V10 increases ${ }^{[8]}$.

IMRT lung studies showed that as the number of radiation fields increases, V5 and V10 in the lungs increase ${ }^{[9]}$. In summary, Options $D(5$ semifield IMRT in the upper and 3 semi-field 3D-CRT in the lower target area) and E (5 semi-field IMRT in the upper and 3 semi-field IMRT in the lower target area) are the superior options. They are as effective as the other techniques in delivering a dose to the target area while minimizing normal tissue exposure to low doses, especially V5, V10, and V20 of Option $\mathrm{D}$ and a lower MLD in Options D and E. Option D has shorter treatment times than Option E. Options $D$ and $E$ have less homogeneity than the others. Junqi Wang, et al. stated that target-area dosehomogeneity in radiotherapy was significant for radiotherapists when evaluating various treatment options. Upper, and middle, esophageal carcinoma tumors with their lower viability and poor prognosis argue that the main complication of radiotherapy radiation pneumonitis - should first be taken into account ${ }^{[10]}$.

\section{ACKNOWLEDGEMENT}

The author thanks Jiutang Zhang, Hui Wang, and Qianxi Ni (Department of Radiotherapy, Hunan Cancer Hospital and the Affiliated Cancer Hospital of Xiangya Medicine, Central South University, Changsha 410013, Hunan, P.R. C.) and Jianhui Liu (Department of Information, Hunan Hospital of Armed Police Forces, Changsha 410006, Hunan, P.R.C.) for advice, assistance, and technical support in sample collection and data acquisition.

This work was supported by a grant from the Science and Technology Program of Hunan 
Provincial Science and Technology Department (program number: 2014SK3086) and National Key Clinical Specialty (Oncology Department) (National Health and Family Planning Commission of the P.R. China 2013/544).

\section{REFERENCES}

1. Cui N, Lu T, Deng X. Practical and clinical radiation oncology. Guangzhou: Sun Yat-sen University Press. 2005.

2. Zeng B, Lu X. The clinical application of intensitymodulated radiotherapy plan system of oncentra masterplan. Med Equipment. 2010; 23(5): 6-8.

3. Hu Y, Zhang H, Dai J. Tumor radiation physics. Beijing: Atomic Energy Press. 1999.

4. Xian J, Zhang L, Li X, Pan X, Jiang C, Wang Y, Li Z. Comparison between IMRT and 3D-CRT in the Therapy of Mid-thoracic Esophageal Cancer. J Military Surg Southwest China. 2011; 4: 2.

5. Chen J, Yong Y, Liu T, Dong X, Wang D. Application of IMAT versus fixed-gantry IMRT in cervical esophageal cancer: A comparison in dosimetry and implementation. Chin J Radiat
Oncol. 2010; 12(5): 429-430.

6. Graham M V, Purdy J A, Emami B, Harms W, Bosch W, Lockett MA, Perez CA. Clinical dosevolume histogram analysis for pneumonitis after 3D treatment for non -small cell lung cancer (NSCLC). Int J Radiat Oncol Biol Phys. 1999; 45(2): 323-329.

7. Wang L, Han C, Zhang X, Wang J, Xiao A, Ma G. The observation of three dimensional conformal radiotherapy curative effect. Chin J Clini Oncol. 2008; 35 (8): 424-427.

8. Schallenkamp JM, Miller RC, Brinkmann DH, Foote T, Garces YI. Incidence of radiation pneumonitis after thoracic irradiation: Dosevolume correlates. Int J Radiat Onco Biol Phys. 2007; 67(2): 410-416.

9. Guo X, Mei X, Zhu G. Comparison of conventional and conformal radiotherapy and different irradiation technique by 3D-TPS for esophageal carcinoma: a dosimetric study. Chin Oncol. 2005; 15(5): 462-465.

10. Wang J, Li L, Xu Z, Yang H, Fu X. Application of dose heterogeneity in the target volume in intensity-modulated radiation therapy of esophageal cancer. Chin J Radiol Med Protect. 2011; 31(4): 453-455. 